\title{
Endocarditis infecciosa por Listeria monocytogenes: revisión de la literatura a partir de un caso clínico
}

\author{
Arnaldo Marín, Nicole Lustig, Ricardo Cruz y Samuel Córdova
}

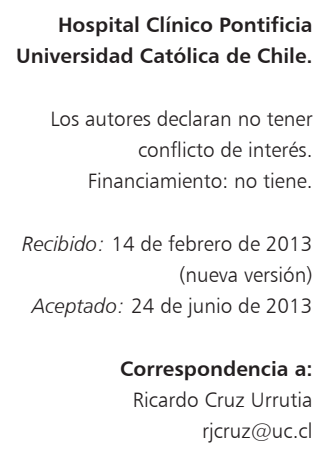

\section{Infective endocarditis caused by Listeria monocytogenes: review of the literature and a case report}

Infective endocarditis caused by Listeria monocytogenes is an extremely rare but usually aggressive disease. We have seen in recent years an increase in age of onset of this disease and a predilection for patients with valvular disease, especially prosthetic valve. The treatment of choice is ampicillin, which is combined with gentamicin for synergy. It is known that even with treatment the mortality is very high and higher than in other types of bacterial endocarditis. With optimal therapy, that is medical-surgical, mortality has declined significantly in recent years. We presents a review of the literature and a clinical case of a patient with a febrile syndrome diagnosed as a infective endocarditis caused by L. monocytogenes treated medically with successful outcome to date.

Key words: Infective endocarditis, Listeria monocytogenes, case report.

Palabras clave: Endocarditis infecciosa, Listeria monocytogenes, caso clínico.

\section{Introducción}

L a endocarditis infecciosa (EI) es una enfermedad grave que afecta al endocardio valvular o mural. Puede instalarse en defectos congénitos o adquiridos, como válvulas o prótesis cardíacas, fístulas arterio-venosas y otras. Su desenlace sin tratamiento antimicrobiano es prácticamente fatal en todos los casos ${ }^{1}$.

En Chile, esta enfermedad tiene una incidencia de 1,7 a 6,2 casos por 100.000 personas-año. En el área central de Santiago se ha registrado una incidencia de dos casos por 100.000 pacientes por año ${ }^{1}$.

Puede ser causada por una variedad de microorganismos (bacterias y hongos). De las bacterias que causan endocarditis destacan Streptococccus sp y Enterococcus sp, apreciándose en el último tiempo un aumento en la incidencia de Staphylococcus sp, bacilos gramnegativos y grampositivos ${ }^{2}$.

Listeria monocytogenes es un bacilo grampositivo no esporulado, aeróbico. Es la única de las especies de Listeria que causa enfermedad en humanos ${ }^{3}$. De los siete serotipos principales, $4 \mathrm{~b}$, 1a y $1 \mathrm{~b}$ son los responsables de la mayoría de las listeriosis humanas ${ }^{4}$.

Listeria monocytogenes causa una serie de cuadros clínicos entre los que se incluyen sepsis, meningoencefalitis y una amplia variedad de localizaciones como artritis, osteomielitis, peritonitis, hepatitis, colecistitis y endocarditis 5 . Afecta preferentemente a recién nacidos y adultos mayores de 60 años, mujeres embarazadas y pacientes con inmunocompromiso (diabetes mellitus, cirrosis hepática, etilismo crónico, tumores sólidos par- ticularmente adenocarcinoma de colon), aunque también puede afectar a personas sanas ${ }^{6-12}$.

Esta bacteria ha sido aislada en una variedad de alimentos ${ }^{12-14}$, sin embargo, la mayoría de los pacientes con endocarditis por L. monocytogenes no tiene historia de exposición a alimentos contaminados.

Se presenta el primer caso clínico en Chile de una endocarditis por L. monocytogenes en un adulto, y una revisión de la literatura científica sobre el manejo y particularidades clínicas de este patógeno en el endocardio valvular.

\section{Caso clínico}

Varón de 77 años, jubilado, que ingresó a nuestro hospital con el diagnóstico de síndrome febril en estudio.

Tenía antecedentes de una coartación aórtica operada, con recambio valvular aórtico hacían ya 40 años, reemplazo compuesto de válvula aórtica y aorta ascendente y un reemplazo valvular mitral, tres años atrás, por lo que se encontraba en tratamiento anticoagulante oral. Además presentaba hipertensión arterial, insuficiencia cardíaca secundaria e hipotiroidismo en control.

Consultó por un mes de evolución de fiebre intermitente cuantificada hasta $38{ }^{\circ} \mathrm{C}$ axilar, asociada a dolor abdominal moderado difuso, de carácter inespecífico, sudoración nocturna y pérdida de peso de $6 \mathrm{~kg}$ en el último mes. Sin diarrea, vómitos, tos, disuria, artralgias ni lesiones cutáneas. Dentro de los antecedentes epidemiológicos, residía en Santiago y no había realizado viajes al extranjero. 
Al momento de la evaluación el paciente se encontraba consciente, vigil, cooperador, con hemodinamia estable, yugulares planas y sin adenopatías palpables. El examen pulmonar era normal, al examen cardíaco presentaba un soplo sistólico en foco aórtico 3/6, el abdomen era blando, depresible, sensible en forma difusa a la palpación, sin signos de irritación peritoneal ni visceromegalia. Las extremidades inferiores no presentaban edema y la piel y lechos ungueales no tenían lesiones. El fondo de ojo fue normal.

Se realizaron exámenes de laboratorio, destacando un examen de orina completa y función renal normales, hemograma con una anemia normocítica normocrómica (hemoglobina de $11 \mathrm{gr} / \mathrm{dL}$ y hematocrito de $31,7 \%$ ), con las otras series normales; VHS $82 \mathrm{~mm} 1^{\circ}$ hora; PCR 5,3 $\mathrm{mg} / \mathrm{dL}(\mathrm{VN}<1 \mathrm{mg} / \mathrm{dL})$ y un INR 2,5 . Por el cuadro de dolor abdominal se solicitaron pruebas hepáticas: GOT 45 UI/L, GGT $81 \mathrm{UI} / \mathrm{L}$, bilirrubina total $0,69 \mathrm{mg} / \mathrm{dL}, \mathrm{LDH}$ 360 U/L, GPT y fosfatasas alcalinas normales. Se realizaron serologías para virus de hepatitis A, B y C y VIH que resultaron negativas, reacción de Widal negativa, endoscopia digestiva alta normal y ecografía abdominal sin alteraciones. Entre otros exámenes destacaba TSH 0,84 $\mathrm{U} / \mathrm{ml}$ (VN 0,3-4,2 U/ml) y factor reumatoide negativo.

Por persistencia del cuadro, fue internado donde se continuó el estudio con una radiografía de tórax AP y lateral que fue normal. Por el antecedente de una válvula protésica se realizó un ecocardiograma transesofágico que mostró una imagen filamentosa de 20 × 8 × $5 \mathrm{~mm}$ en la válvula aórtica (Figuras 1 y 2).

Se inició tratamiento empírico para una EI asociada a válvula protésica con vancomicina iv $1 \mathrm{~g}$, dos veces al día, gentamicina iv $80 \mathrm{mg}$, tres veces al día y rifampicina 600 $\mathrm{mg}$ al día. Los dos hemocultivos tomados previo al inicio de tratamiento antibacteriano, fueron positivos a las 23 hrs. a un bacilo grampositivo, que fue identificado como $L$. monocytogenes. Se ajustó el tratamiento a ampicilina $2 \mathrm{~g}$ iv, seis veces al día y gentamicina $80 \mathrm{mg}$, tres veces al día.

Por presentar una buena respuesta clínica se decidió el alta y continuó con tratamiento antibacteriano intravenoso en forma ambulatoria. Se controló en forma mensual durante los tres primeros meses, luego cada tres meses hasta completar un año y posteriormente cada seis meses durante el segundo año. Durante su seguimiento se constató un descenso de los parámetros inflamatorios y se ajustaron las dosis de los antibacterianos según su función renal. Completó tres semanas de tratamiento con gentamicina $80 \mathrm{mg}$, dos veces al día, suspendiéndose por deterioro de la función renal; ampicilina $2 \mathrm{~g}$, cuatro veces al día, por doce semanas y posteriormente recibió tratamiento de mantención con amoxicilina $1 \mathrm{~g}$, dos veces al día y y luego terapia supresora con $500 \mathrm{mg}$ al día, hasta completar dos años, para garantizar la esterilidad de la lesión residual. El último ecocardiograma de control

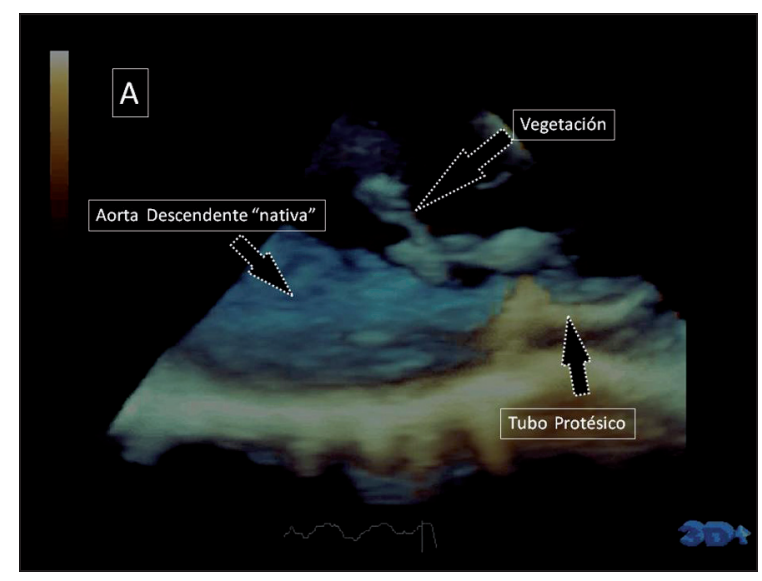

Figura 1. Ecocardiograma trans-esofágico 3D: imagen de la aorta descendente, destacándose la unión entre el margen distal del tubo protésico (instalado en la reparación de la coartación) y la aorta torácica descendente nativa. Vegetación en "rosario" de 30 × $8 \mathrm{~mm}$ en el margen del tubo protésico.

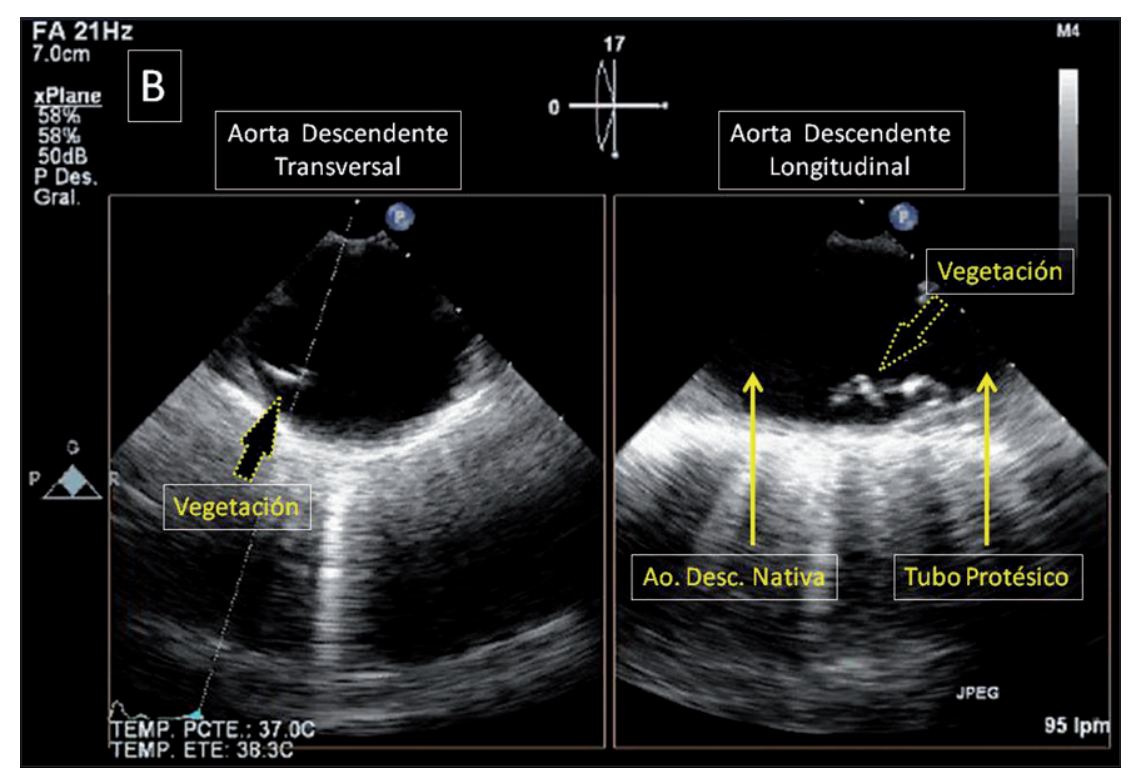

Figura 2. Ecocardiograma trans-esofágico 2D: dos cortes tranversal y longitudinal de la aorta torácica descendente a nivel de la unión distal del tubo protésico y la aorta torácica descendente. Vegetación del tubo protésico.

no presentaba variaciones significativas con respecto al examen inicial. Actualmente, el paciente se ha mantenido asintomático.

\section{Discusión}

La endocarditis infecciosa (EI) por L. monocytogenes es una enfermedad extremadamente infrecuente, pero usualmente agresiva debido a su capacidad de escapar a la detección inmune ${ }^{14}$. En 1955 fue publicado el primer caso en la literatura internacional ${ }^{15}$. Hasta la fecha se ha comunicado un total de 68 casos de EI por L. monocytogenes ${ }^{16}$.

Desde el punto de vista epidemiológico destacan tres aspectos: un aumento de la edad de presentación de 47 
Tabla 1. Comparación de endocarditis infecciosa por Listeria monocytogenes en válvula nativa vs válvula protésica

\begin{tabular}{|c|c|c|}
\hline Variable & $\begin{array}{l}\text { Válvula nativa } \\
\text { ( } n=45 \text { pacientes) }\end{array}$ & $\begin{array}{l}\text { Válvula protésica } \\
\text { ( } n=23 \text { pacientes) }\end{array}$ \\
\hline Promedio de edad & 50 años & 65,3 años \\
\hline Hombres & $58,3 \%$ & $80 \%$ \\
\hline Condiciones no cardíacas subyacentes & $51,1 \%$ & $30,4 \%$ \\
\hline Condiciones cardíacas subyacentes & $53,3 \% *$ & $100 \%$ \\
\hline \multicolumn{3}{|l|}{ Válvula } \\
\hline Mitral & $15,6 \%$ & $52,2 \%$ \\
\hline Aórtica & $26,7 \%$ & $34,8 \%$ \\
\hline Mitro-aórtico & $31,1 \%$ & $13 \%$ \\
\hline Tricúspide & $4,4 \%$ & - \\
\hline Tricúspide-aórtico & $2,2 \%$ & - \\
\hline No indicado & $20 \%$ & - \\
\hline Mortalidad global & $44,4 \%$ & $21,7 \%$ \\
\hline \multicolumn{3}{|l|}{ Tratamiento** } \\
\hline Sólo médico & $81,1 \%$ & $50 \%$ \\
\hline Mortalidad & $40 \%$ & $36,4 \%$ \\
\hline Quirúrgico & $18,8 \%$ & $50 \%$ \\
\hline Mortalidad & $42,9 \%$ & $9,1 \%$ \\
\hline \multicolumn{3}{|c|}{$\begin{array}{l}\text { *15 de los } 45 \text { pacientes }(33,3 \%) \text { con válvula nativa con endocarditis tenían un daño valvular previo. } \\
\text { **El tratamiento total fue completamente conocido en un total de } 59 \text { pacientes. Adaptado de Fer- } \\
\text { nández Guerrero ML. y cols. }{ }^{16}\end{array}$} \\
\hline
\end{tabular}

años a 65 años, cerca de $60 \%$ de los pacientes tenía alguna enfermedad valvular, de los cuales 33,8\% tenía una válvula protésica y por último se ha observado un aumento en los casos de EI por L. monocytogenes en pacientes con válvula protésica. Entre 1955 y 1984, 18\% de las endocarditis por Listeria sp. afectaban válvulas protésicas, mientras que entre 1985 y 2000 aumentó a 53\% ${ }^{16}$.

Existen algunas alteraciones predisponentes a desarrollar una EI entre las que se encuentran la valvulopatía reumática, cardiomiopatía hipertrófica, prolapso mitral y cardiomiopatía isquémica ${ }^{17-22}$.

La endocarditis por L. monocytogenes a menudo ocurre en una válvula nativa dañada o protésica, principalmente en la aórtica, mitral o ambas, siendo infrecuente en la válvula tricúspide ${ }^{23-26}$. Se han descrito diferencias clínicas y en el pronóstico de pacientes con endocarditis por $L$. monocytogenes, según si existe compromiso de una válvula nativa o protésica. En un estudio comparativo se demostró que la mayoría de los pacientes eran de sexo masculino, con patologías cardíacas previas y localizadas en válvulas de la circulación mayor. La mortalidad fue mayor en los pacientes con válvulas nativas y en aquellos que sólo recibieron tratamiento médico ${ }^{16}$ (Tabla 1).

Los hallazgos patológicos pueden ser vegetaciones, destrucción o dehiscencia de la prótesis y en ocasiones la formación de abscesos miocárdicos, fistulización y pericarditis $^{11,27}$.
Su curso clínico usualmente es de carácter subagudo caracterizado por fiebre, síntomas constitucionales y manifestaciones cardíacas ${ }^{17,18,28-34}$ destacando la insuficiencia cardíaca en $65 \%$ de los casos $^{35}$.

El tratamiento óptimo de la endocarditis por L. monocytogenes no ha sido completamente consensuado. Esta bacteria es susceptible a una amplia variedad de antibacterianos y es poco frecuente encontrar resistencia ${ }^{36}$. Cuando ésta ocurre es debido a elementos de transferencia genética de Enterococcus sp. o Streptococcus sp. a L. monocytogenes lo que podría determinar resistencia a macrólidos, lincosamidas/estreptograminas y tetraciclina ${ }^{36,37}$ . En forma infrecuente se han reportado cepas resistentes a gentamicina, estreptomicina y cotrimoxazol ${ }^{38,39}$. Las cefalosporinas de tercera generación tienen una actividad débil contra L. monocytogenes debido a una baja afinidad a las PBP-3 (penicillin binding protein) ${ }^{39,40}$. Listeria monocytogenes es sensible a penicilina, vancomicina, cotrimoxazol y algunas quinolonas ${ }^{27,40}$. Ampicilina es el antibacteriano de elección para su tratamiento ${ }^{28,41}$, la que es combinada con gentamicina para obtener sinergismo. Otra combinación posible es vancomicina asociada a gentamicina.

Se recomienda que el tratamiento para la endocarditis por L. monocytogenes debiera tener una duración mínima de 4 semanas con válvula nativa y 6 a 8 semanas con válvula protésica.

El pronóstico de esta enfermedad, con tratamiento adecuado, tiene una mortalidad de $20-30 \%$ en series internacionales, comparada con $14 \%$ de los otros tipos de endocarditis bacteriana ${ }^{16}$; sin embargo, la mortalidad en la última década ha tendido a disminuir. Esto podría deberse a una disminución del umbral para el tratamiento quirúrgico. Aunque muchos pacientes, incluidos los con válvula protésica, fueron curados sólo con antimicrobianos, la combinación de un tratamiento médico-quirúrgico parece reducir la mortalidad en pacientes con endocarditis por L. monocytogenes ${ }^{28-32,42-46}$. Las indicaciones de cirugía son las mismas que para otras formas de endocarditis ${ }^{46}$.

\section{Conclusión}

La endocarditis infecciosa por L. monocytogenes es una enfermedad poco común pero de evolución grave, que ocurre a menudo sobre una válvula disfuncionante y puede generar insuficiencia cardíaca secundaria.

El tratamiento actual recomendado es ampicilina o su combinación con gentamicina.

A pesar de los problemas asociados con la resistencia y recaídas en algunas formas de listeriosis humana, la terapia antimicrobiana por si sola puede ser útil para el tratamiento de la endocarditis por Listeria sp. incluyendo los casos que ocurren en válvulas protésicas. La terapia óptima corresponde a un tratamiento antibacteriano y 
cirugía oportuna lo que ha logrado disminuir significativamente la mortalidad en los últimos años. Se sabe que aún con un tratamiento adecuado, la mortalidad es de $20-30 \%$ comparada con $14 \%$ de mortalidad de todos los tipos de endocarditis infecciosa.

Se presenta un caso clínico que tuvo una evolución favorable sólo con tratamiento médico. En este caso no se detectó una fuente de contagio específica, pero se identificaron múltiples factores de riesgo cardiovasculares estructurales (prótesis), para desarrollar la endocarditis infecciosa.

\section{Resumen}

La endocarditis infecciosa por Listeria monocytogenes es una enfermedad extremadamente infrecuente, pero usualmente agresiva. Se ha observado en los últimos años un aumento en la edad de presentación y una predilección por pacientes con enfermedad valvular, especialmente válvula protésica. El tratamiento de elección es ampicilina combinada con un aminoglucósido para obtener sinergismo. Aun con un tratamiento adecuado, la mortalidad es elevada y mayor que en otros tipos de endocarditis bacteriana. Con una terapia óptima, incluyendo la resolución quirúrgica, la mortalidad ha disminuido significativamente en los últimos años. Se presenta una revisión de la literatura científica a partir de un caso clínico de un paciente con un síndrome febril en el que se diagnosticó una endocarditis por L. monocytogenes que fue tratada en forma médica con un resultado exitoso.

\section{Referencias bibliográficas}

1.- Oyonarte M, Montagna R, Braun S, Maiers E, Rojo P, Cumsille J F. Endocarditis infecciosa: morbimortalidad en Chile. Resultados del Estudio Cooperativo Nacional de Endocarditis Infecciosa (1998-2002). Rev Med Chile 2003; 131: $237-50$

2.- Oyonarte M, Braun S, Maiers E, Lueg E, Montagna R. Guías Clínicas de la Sociedad Chilena de Cardiología y Cirugía Cardiovascular para el estudio y manejo de la endocarditis infecciosa. Rev Chil Cardiol 2004; 1: 41-52.

3.- Lorber B. Listeriosis. Clin Infect Dis 1997; 24: 10-1.

4.- Rossi M L, Paiva A, Tornese M, Chianelli S, Troncoso A. Brotes de infección por Listeria monocytogenes: Una revisión de las vías que llevan a su aparición. Rev Chilena Infectol 2008; 25: 328-35.

5.- Karavidas A, Halapas A, Zacharoulis A, Matsakas E, Zacharoulis A. A subacute bacterial endocarditis in a patient with aortic prosthetic valve due to Listeria monocytogenes presenting with perivalvular leak. Int J Cardiol 2007; 118: e106-7.

6.- Carvajal A, Frederiksen W. Fatal endocarditis due to Listeria monocytogenes. Rev Infect Dis 1988; 10: 616-23.

7.- Goulet V, Marchetti P. Listeriosis in 225 nonpregnant patients in 1992: clinical aspects and outcome in relation to predisposing conditions. Scand J Infect Dis 1996; 28: 367-74.

8.- Schlech W F $3^{\text {rd }}$. Foodborne listeriosis. Clin Infect Dis 2000; 31: 770-5.

9.- Julián A, Jiménez A, de Górgolas M, Fernández R, Fernández M. Infecciones por Listeria monocytogenes en el adulto. Aspectos clínicos y microbiológicos de una enfermedad cambiante. Enferm Infecc Microbiol Clin 2001; 19: 297-303.

10.- Segura J, Anguita M, Vivancos R, Franco M,
Romo E, Suárez de Lezo J, et al. Listeria monocytogenes endocarditis in a patient with mitral prosthesis, left auricular thrombus and adenocarcinoma of the colon. Rev Esp Cardiol 1992; 45: 483-5.

11.- Castro Cabezas M, Cramer M J, de Jongh B M, de Maat CE. Listeria monocytogenes endocarditis in a patient with an aortic prosthetic valve. Neth J Med 1996; 48: 15-7.

12.- Taege A J. Listeriosis: recognizing it, treating it, preventing it. Cleve Clin J Med 1999; 66: 37580.

13.- Schuchat A, Deaver K A, Wenger J D, Plikaytis BD, Mascola L, Pinner RW, et al. Role of foods in sporadic listeriosis. I. Case-control study of dietary risk factors. JAMA 1992; 267: 2041-5.

14.- Pinner R W, Schuchat A, Swaminathan B, Hayes P S, Deaver K, Weaver R E, et al. Role of foods in sporadic listeriosis. II. Microbiologic and epidemiologic investigation. JAMA 1992; 267: 2046-50.

15.- Hoeprich P D, Chernoff H M. Subacute bacterial endocarditis due to Listeria monocytogenes. Am J Med 1955; 19: 488-94.

16.- Fernández Guerrero M L, Rivas $P$, Rábago R, Núñez A, de Górgolas M, Martinell J. Prosthetic valve endocarditis due to Listeria monocytogenes. Report of two cases and reviews. Int J Inf Dis 2004; 8: 97-102.

17.- Brecht W, Oesterle D. Uber endocarditis listeriosa. Chemotherapia 1962; 4: 573-9.

18.- Clark RA. Bacterial endocarditis caused by Listeria monocytogenes. West J Med 1977; 126: 403-5.

19.- Pitcher D, Mary D. Listeria monocytogenes endocarditis in hypertrophic cardiomyopathy. $\mathrm{Br}$ Med J 1978; 1 (6118): 961.

20.- Winslow D, Scott E. Listeria sepsis: a report of 27 cases. Infect Dis Rev 1981; 6: 187-215.

21.- Gallagher P G, Watanakunakorn C. Listeria monocytogenes endocarditis: a review of the literature. Scand J Infect Dis 1988; 20: 359-68.
22.- Riancho J A, Echevarría S, Napal J, Martin Durán R, González Macías J. Endocarditis due to Listeria monocytogenes and human immunodeficiency virus infection. Am J Med 1988; 85: 737

23.- Leonard A, Raij L, Shapiro F L. Bacterial endocarditis in regularly dialyzed patients. Kidney Int 1973; 4: 407-22.

24.- Speeleveld E, Muyldermans L, Van den Bruel A, Dhondt E, Gordts B, Van Landuyt H W. Prosthetic valve endocarditis due to Listeria monocytogenes. A case report with review of the literature. Acta Clin Belg 1994; 49: 95-8.

25.- Manso C, Rivas I, Peraire J, Vidal F, Richart C. Fatal Listeria meningitis, endocarditis and pericarditis in a patient with haemochromatosis. Scand J Infect Dis 1997; 29: 308-9.

26.- Avery R K, Barnes D S, Teran J C, Wiedemann H P, Hall G, Wacker T, et al. Listeria monocytogenes tricuspid valve endocarditis with septic pulmonary emboli in a liver transplant recipient. Transpl Infect Dis 1999; 1: 284-7.

27.- Hood S, Baxter R H. Listeria endocarditis causing aortic root abscess and a fistula to the left atrium. Scott Med J 1999; 44: 117-8.

28.- Spyrou N, Anderson M, Foale R. Listeria endocarditis: current management and patient outcome-world literature review. Heart 1997; 77: 380-3.

29.- Elston H R, Zencka A E, Sketch M H. Listeria monocytogenes endocarditis: a clinical and bacteriologic report. Arch Intern Med 1969; 124: 488-91.

30.- De Soldati L, Alescio A C, Cola H J. Bacterial endocarditis due to Listeria monocytogenes. Cardiology 1972; 57: 172-80.

31.- Bayer A S, Chow A W, Guze L B. Listeria monocytogenes endocarditis: report of a case and review of the literature. Am J Med Sci 1977; 273: 319-23.

32.- Kaplan J, Stollenwerk A. Listeria 
monocytogenes endocarditis. Arch Intern Med 1977; 137: 1484.

33.- Holshouser C A, Ansbacher R, McNitt T, Steele R. Bacterial endocarditis due to Listeria monocytogenes in a pregnant diabetic. Obstet Gynecol 1978; 51: 9s-10s.

34.- Breyer R H, Arnett E N, Spray T L, Roberts W C. Prosthetic-valve endocarditis due to Listeria monocytogenes. Am J Clin Pathol 1978; 69: 186-7

35.- Savalolatz L D, Burch K H, Madhavan T, Quinn E L. Listerial prosthetic valve endocarditis. Successful medical therapy. JAMA 1978; 240: 2186-7.

36.- Charpentier E, Courvalin P. Antibiotic resistance in Listeria spp. Antimicrob Agents Chemother 1999; 43: 2103-8

37.- Hadorn K, Hachler H, Schaffner A, Kayser F H. Genetic characterization of plasmid-encoded multiple antibiotic resistance in a strain of Listeria monocytogenes causing endocarditis. Eur J Clin Microbiol Infect Dis 1993; 12: 928-37.

38.- McGowen A P, Reeves D S, McLauchlin J. Antibiotic resistance of Listeria monocytogenes. Lancet 1990; 336: 513-4.

39.- Tsakaris A, Papa A, Douboyas J, Antoniadis A. Neonatal meningitis due to multi-resistant Listeria monocytogenes. J Antimicrob Chemother 1997; 39: 553-4.

40.- Vicente M F, Pérez-Dáz J C, Baquero F, Angel de Pedro M, Berenguer J. Penicillinbinding protein 3 of Listeria monocytogenes as the primary lethal target for beta-lactams. Antimicrob Agents Chemother 1990; 34 : $539-42$.

41.- Hof H, Nichterlein T, Kretschmar M. Management of listeriosis. Clin Microbiol Rev
1997; 10: 345-57

42.- Torres L, Blasco M, Marco M L, Ruiz M A, Uriel B, Revillo P. Listeria monocytogenes endocarditis of a prosthetic valve. Rev Esp Cardiol 2000; 53: 300 .

43.- Goasguen J, Costeseque P, Josserand C, Oddou A, Paillet R, Sagnet H. Septicemia with endocarditis caused by Listeria monocytogenes in a 10-year-old Biafran child. Med Trop 1969; 29: 699-701.

44.- Higgins T L, Mallek J A, Slugg P H. Listeria monocytogenes endocarditis on a prosthetic heart valve. South Med J 1983; 76: 675-6.

45.- Davis W R, Taylor J L. Listeria monocytogenes prosthetic valve endocarditis. South Med J 1983; 76: 1074

46.- Brouqui P, Raoult D. Endocarditis due to rare and fastidious bacteria. Clin Microbiol Rev 2001; 14: 177-207. 